Article

\title{
Design of a Low-Order FIR Filter for a High-Frequency Square-Wave Voltage Injection Method of the PMLSM Used in Maglev Train
}

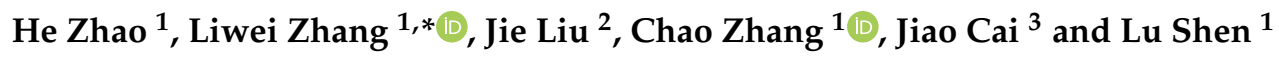 \\ 1 School of Electrical Engineering, Beijing Jiaotong University, Beijing 100044, China; \\ 17121540@bjtu.edu.cn (H.Z.); 18117026@bjtu.edu.cn (C.Z.); 19117017@bjtu.edu.cn (L.S.) \\ 2 Anhui Huaying Automotive Technology Co., Ltd., Anhui 239400, China; liujiebjh@dingtalk.com \\ 3 Information Technology Center, Beijing Jiaotong University, Beijing 100044, China; caijiao@bjtu.edu.cn \\ * Correspondence: lwzhang@bjtu.edu.cn; +86-135-5268-3918
}

Received: 4 April 2020; Accepted: 26 April 2020; Published: 28 April 2020

\begin{abstract}
In position sensorless control based on a high-frequency pulsating voltage injection method, filters are used to complete the extraction of high-frequency response signals for position observation. A finite impulse response (FIR) filter has the advantages of good stability and linear phase. However, the FIR filter designed by using traditional methods has a high order which will cause a large time delay. This paper proposes a low-order FIR filter design method for a high-frequency signal injection method in the permanent magnet linear synchronous motor. Based on the frequency characteristics of the current signal, the requirement that the FIR filter needs to meet were analyzed. According to the amplitude-frequency characteristic of the FIR filter, these requirements were converted into constraint equations. By solving these equations, the coefficient of the FIR filter could be obtained. The simulation and experiment results showed the effectiveness of this low-order FIR filter.
\end{abstract}

Keywords: PMLSM; position sensorless control; high-frequency square-wave voltage injection; FIR filter; maglev train

\section{Introduction}

Due to the fact of its high efficiency and high-power density, the permanent magnet linear synchronous motor (PMLSM) is used in the drive system of the maglev train. For the PMLSM control system, position detection is crucial. There are many traditional position detection methods [1-5]. The inductive looped-cable method was used in References [1-3] which requires signal process units, crossed-looped cables, and antennas. In addition, the crossed-looped cables need to be placed along the rails which will make the system very expensive to construct and maintain [4]. In References [4,5], radio millimeter waves and Doppler radar are used to detect the position of the maglev train. Although they do not need to lay lines, they need additional equipment and design such as radar and mobile, base, and central stations. This will also increase the cost and the complexity of mechanical installation [6]. Therefore, the position sensorless control method has received increasing research attention.

Position sensorless control can be classified into two types. The first type is based on the back electromotive force (EMF) of the motor [7]. To improve the performance of the position sensorless control system, the extended back EMF method [8], sliding mode observer [9], and extended Kalman filter [10] are used in this method. However, when the motor is at zero or low speed, the back EMF is too small to detect which means this method is not applicable. The second type is based on motor saliency which can realize position estimation at zero or low speed, and the high-frequency signal injection method is representative of this type. Currently, research on the high-frequency signal injection method 
is mainly concentrated on the permanent magnet synchronous motor (PMSM). As the PMLSM and PMSM have the same working principles and similar mathematical models, these high-frequency signal injection methods can be directly used for PMLSM. Injecting a high-frequency rotating voltage into a stationary reference frame and injecting a high-frequency pulsating voltage into an estimated synchronous reference frame are two traditional methods of high-frequency signal injection method [11]. In addition, after years of research, many improvements have appeared [12-16]. In Reference [12], the high-frequency pulsating signal was injected into the estimated fixed-frequency rotating reference frame instead of the estimated synchronous reference frame. In addition, the high-frequency pulsating signal was injected into the stationary reference frame in Reference [13] and was injected into the ABC reference frame in Reference [14]. In Reference [15], a bidirectional rotating signal was used, and a high-frequency square-wave voltage signal was used in Reference [16]. The differences among these methods lay in the form of the injection signal and the selection of the injection reference frame. However, all methods are required in order to extract the high-frequency response signal which carries magnetic pole position information from the motor current. This extraction process directly determines the accuracy of the position estimation.

In the PMLSM control system, the inverter will induce a large amount of harmonics. When the high-frequency square-wave voltage injection method is used to realize position estimation, these harmonics will affect the estimation accuracy [16]. Increasing the amplitude of injection voltage can increase the signal-to-noise ratio (SNR) which can reduce the effect of harmonics. However, the voltage utilization will be reduced [17]. Moreover, when PMLSM is used in the maglev train drive system and the high-frequency square-wave voltage is injected, the current will fluctuate and generate normal force fluctuation. This means that increasing the amplitude of injection voltage will increase the impact on the suspension system. In addition to increasing the amplitude of injection voltage, digital filters can also help to improve the SNR. The infinite impulse response (IIR) filters were designed in References [18,19]. However, the IIR filter is a nonlinear phase filter and requires output feedback which increases the complexity of the control system. Furthermore, according to the research in Reference [20], the FIR filter has a better performance than the IIR filter in the position estimation system. An FIR filter was designed in Reference [21], but its order is as high as 17 which will cause a large time delay. A moving average filter was designed in Reference [22] which can provide the system with a higher bandwidth and better harmonics suppression capability. But it is also an FIR filter with a high order of 21. In order to reduce the time delay, many studies have proposed different improvements. An algebraic operation was used in References $[23,24]$ to separate carrier signals, but this calculation process is ideal without considering the influence of harmonics. A direct signal demodulation method was proposed in Reference [25] which can remove the low-pass filter, but it is mainly used in the initial position estimation. Reference [26] designed an all-pass filter to calculate the position which can eliminate the time delay caused by the band-pass filter and low-pass filter, but the nonlinearity of the inverter is not considered either.

After considering the influence of the inverter and the time delay caused by the filter, this paper proposes a low-order FIR filter design method. Different from the traditional design method, this new method does not need to determine the passband and stopband of the filter. Based on the analysis of the motor current components, the amplitude-frequency characteristics at some special frequencies were mainly considered. Compared with the traditional design method, this new method can reduce the order of the FIR filter which will reduce the time delay. Moreover, compared with the filterless estimation system, the amplitude of the injection voltage can be reduced which will increase the voltage utilization [17] and reduce the normal force fluctuation.

\section{Basic Principles of High-Frequency Square-Wave Voltage Injection Method}

The high-frequency square-wave voltage injection method is based on the saliency of the PMLSM. In this paper, a surface-mounted motor was used in the experiment, so the saliency was saturated saliency. The generation of saturated saliency comes from the characteristics of the magnetization 
curve of silicon steel. As the magnetic field strength $\mathrm{H}$ increases, the magnetic field in the silicon steel gradually saturates, and the rising rate of the magnetic induction $\mathrm{B}$ becomes slower. According to the definition of magnetic permeability, $\mu=\mathrm{B} / \mathrm{H}, \mu$ also becomes smaller as $\mathrm{H}$ increases, thereby reducing the inductance. Since the permanent magnet is in the $d$-axis magnetic circuit, the $d$-axis inductance will be less than the $q$-axis inductance at saturation.

By injecting a high-frequency square-wave signal into the virtual $d$-axis, the current of PMLSM will carry the high-frequency signal that contains position information of the magnetic pole. By extracting this high-frequency current signal, the position estimation can be realized.

The voltage equation of PMLSM can be described in the $d-q$ synchronization reference frame (SRF) as shown in Equation (1) [27].

$$
\left[\begin{array}{l}
u_{d} \\
u_{q}
\end{array}\right]=\left[\begin{array}{cc}
R+p L_{d} & -\frac{\pi v}{\tau} L_{q} \\
\frac{\pi v}{\tau} L_{d} & R+p L_{q}
\end{array}\right]\left[\begin{array}{l}
i_{d} \\
i_{q}
\end{array}\right]+\frac{\pi v}{\tau}\left[\begin{array}{c}
0 \\
\psi_{f}
\end{array}\right]
$$

where $u_{d}, u_{q}, i_{d}, i_{q}, L_{d}, L_{q}$ are $d$-axis voltage, $q$-axis voltage, $d$-axis current, $q$-axis current, $d$-axis inductance, and $q$-axis inductance, respectively. And $R, v, \tau, \psi_{f}$, and $p$ are the resistance of motor winding, speed, pole pitch, flux linkage of the permanent magnet, and differential operator, respectively.

With high-frequency excitation, Equation (1) can be simplified. The frequency of the injected square-wave voltage is very high, which means the voltage drop in the resistor is much smaller than the voltage drop in the motor inductor. Therefore, the voltage drop in the resistor can be ignored. In addition, the high-frequency square-wave voltage injection method is used when PMLSM is at low speed, which means speed $v$ is approximately 0 . Therefore, the parts related to $v$ can be also ignored in Equation (1). After simplification, Equation (1) can be expressed as:

$$
\left[\begin{array}{l}
u_{d h} \\
u_{q h}
\end{array}\right]=\left[\begin{array}{cc}
p L_{d} & \\
& p L_{q}
\end{array}\right]\left[\begin{array}{c}
i_{d h} \\
i_{q h}
\end{array}\right] \Rightarrow \frac{d}{d t}\left[\begin{array}{c}
i_{d h} \\
i_{q h}
\end{array}\right]=\left[\begin{array}{cc}
\frac{1}{L_{d}} & \\
& \frac{1}{L_{q}}
\end{array}\right]\left[\begin{array}{l}
u_{d h} \\
u_{q h}
\end{array}\right]
$$

where $i_{d h}, i_{q h}, u_{d h}, u_{q h}$, denote $d, q$ components of high-frequency response current and $d, q$ components of high-frequency injection voltage in SRF, respectively.

Because PMSM and PMLSM generate the three-phase synthetic magnetic field in the same way, the working principle of them are the same. In addition, vector control was used based on the rotating reference frame. Therefore, PMLSM can be analyzed using a rotating reference frame just like PMSM. In order to achieve this goal, the $d-q$ axis in PMLSM was converted to a rotating reference frame in Figure 1. In addition, the $\hat{d}-\hat{q}$ reference frame is also defined in Figure 1 which represents the estimated magnetic pole position. $\Delta \theta=\Delta x / \tau^{*} \pi$ indicates the estimation error which was converted to the rotating reference frame, and $\omega_{r}=v / \tau^{*} \pi$ indicates the motor speed which was converted to angular velocity.

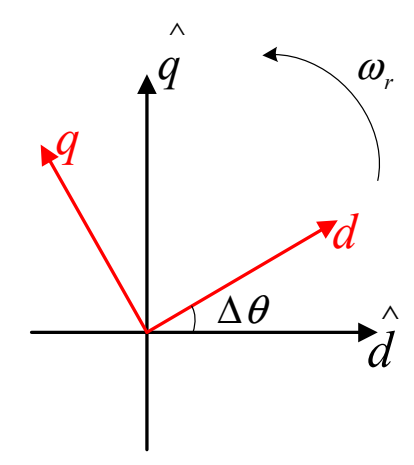

Figure 1. Relationship of the two reference frames. 
The variables in the two coordinates shown in Figure 1 can be converted to each other via Equation (3).

$$
\left[\begin{array}{l}
x_{d} \\
x_{q}
\end{array}\right]=H_{t}\left[\begin{array}{c}
x_{\hat{d}} \\
x_{\hat{q}}
\end{array}\right]=\left[\begin{array}{cc}
\cos \Delta \theta & \sin \Delta \theta \\
-\sin \Delta \theta & \cos \Delta \theta
\end{array}\right]\left[\begin{array}{l}
x_{\hat{d}} \\
x_{\hat{q}}
\end{array}\right]
$$

where $x_{d}$ and $x_{q}$ denote the physical quantities in the $d-q$ reference frame and $x_{\hat{d}}$ and $x_{\hat{q}}$ denote the physical quantities in the $\hat{d}-\hat{q}$ reference frame. $H_{t}$ is the transformation matrix.

By using the coordinate transformation in (3), Equation (2) can be derived as:

$$
\frac{d}{d t}\left[\begin{array}{c}
i_{\hat{d} h} \\
i_{\hat{q} h}
\end{array}\right]=H_{t}^{-1}\left[\begin{array}{cc}
\frac{1}{L_{d}} & \\
& \frac{1}{L_{q}}
\end{array}\right] H_{t}\left[\begin{array}{c}
u_{\hat{d} h} \\
u_{\hat{q} h}
\end{array}\right]=\frac{1}{\sum L^{2}-\Delta L^{2}} \times\left[\begin{array}{cc}
\sum L+\Delta L \cos 2 \Delta \theta & \Delta L \sin 2 \Delta \theta \\
\Delta L \sin 2 \Delta \theta & \sum L-\Delta L \cos 2 \Delta \theta
\end{array}\right]\left[\begin{array}{l}
u_{\hat{d} h} \\
u_{\hat{q} h}
\end{array}\right]
$$

where $\sum L=\left(L_{d}+L_{q}\right) / 2$ and $\Delta L=\left(L_{q}-L_{d}\right) / 2$.

A high-frequency square-wave voltage, which is shown in Figure 2, was injected into the virtual $d$-axis. Its magnitude and period were $V_{h}$ and $T_{h}$, respectively. Due to the simple form of the square wave signal, its frequency could be increased to the inverter switching frequency level. From the above analysis, it can be seen that under high-frequency excitation, the influence of resistance is ignored, and the model of the motor is simplified. The effect of resistance decreases as the frequency of the injected signal increases. Therefore, when the high-frequency square-wave signal injection method is used, it is beneficial to simplify the high-frequency model of PMLSM. Moreover, as the signal frequency increases, the saliency ratio of the motor increases [28] which can enhance the performance of position sensorless control based on saliency.

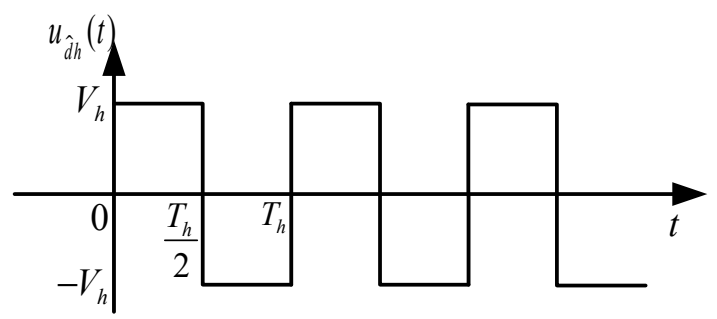

Figure 2. High-frequency square-wave voltage signal diagram.

This injected signal can be expressed as:

$$
\left[\begin{array}{c}
u_{\hat{d} h} \\
u_{\hat{q} h}
\end{array}\right]=\left[\begin{array}{c}
V_{h}(-1)^{k} \\
0
\end{array}\right](k=0,1,2,3 \ldots)
$$

From Equations (4) and (5), the high-frequency current response can be derived as:

$$
\frac{d}{d t}\left[\begin{array}{c}
i_{\hat{d} h} \\
i_{\hat{q} h}
\end{array}\right]=\frac{V_{h}(-1)^{k}}{\sum L^{2}-\Delta L^{2}}\left[\begin{array}{c}
\sum L+\Delta L \cos 2 \Delta \theta \\
\Delta L \sin 2 \Delta \theta
\end{array}\right]
$$

It can be seen from Equation (6) that the high-frequency response current includes $\Delta \theta$ which is the difference between the estimated value and actual value of the magnetic pole position. In order to get the actual position, an observer is needed, as Figure 3 shows.

In Figure $3, i_{\hat{q} f i r}$ is the filtered current of $i_{\hat{q}}$ which is used for current loop feedback. $i_{\hat{q} h}$ is the high-frequency response current caused by injection voltage. It will be eliminated by the FIR filter at first, and then it will be obtained by signal processing. Subsequently, the differential of $i_{\hat{q} h}$ is multiplied by $(-1)^{k}$. The result is shown below.

$$
f(\Delta \theta)=\Delta i_{\hat{q} h} \times(-1)^{k}=\frac{V_{h} \Delta L T_{S}}{\sum L^{2}-\Delta L^{2}} \sin (2 \Delta \theta)
$$




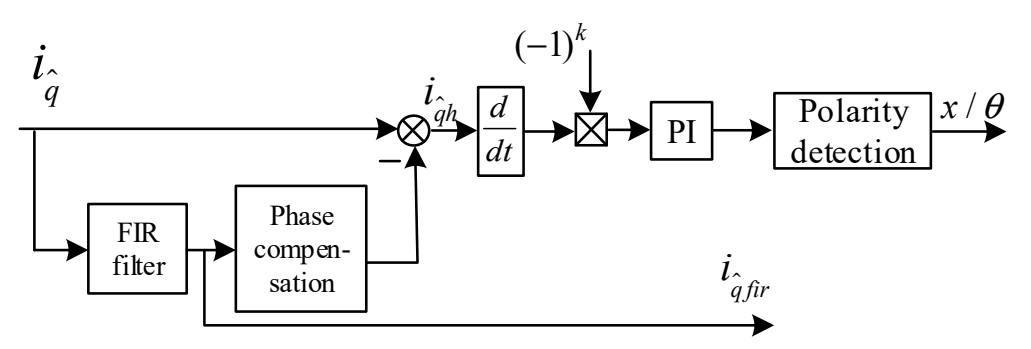

Figure 3. Structure of the position observer.

Finally, the processed signal passes through the PI regulator to change the estimated angle $\hat{\theta}$ so that $f(\Delta \theta)$ becomes 0 . At this time, $\Delta \theta$ will converge to 0 or $\pi$ which means the actual position of the magnetic pole coincides with the estimated position or differs by one pole distance. Therefore, in order to obtain an accurate magnetic pole position, polarity detection is necessary. This paper used the $d$-axis current peak value comparison method to achieve this goal. Figure 4 shows the basic principle of this method. When $\Delta \theta$ converges to 0 , the positive $d$-axis current will increase the magnetic field. At this time, the saturation of the $d$-axis magnetic circuit is increased, so the $d$-axis inductance is reduced. Therefore, the positive current peak $\left|i_{\text {dmax }}\right|$ will be greater than the absolute value of negative current peak $\left|i_{d \min }\right|$. Conversely, when $\Delta \theta$ converges to $\pi$, the negative $d$-axis current will increase the magnetic field which means $\left|i_{d \max }\right|$ will be smaller than $\left|i_{d m i n}\right|$. Therefore, by comparing $\left|i_{d \max }\right|$ and $\left|i_{\text {dmin }}\right|$, the magnetic pole polarity can be detected.

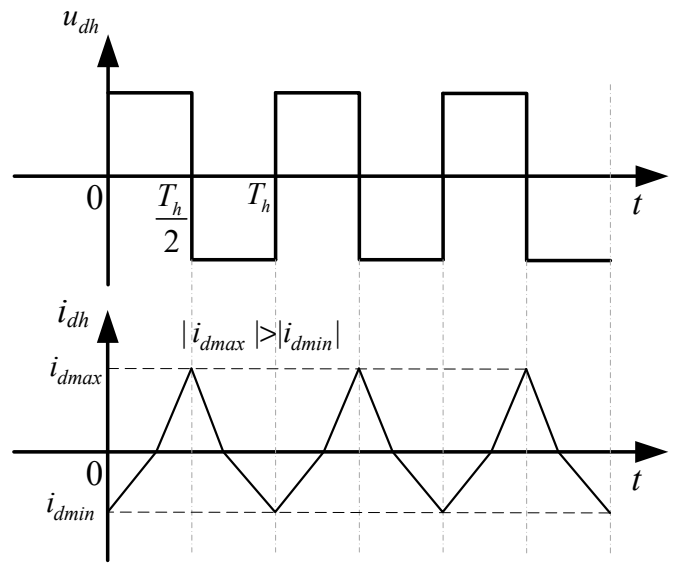

(a) $\Delta \theta=0$

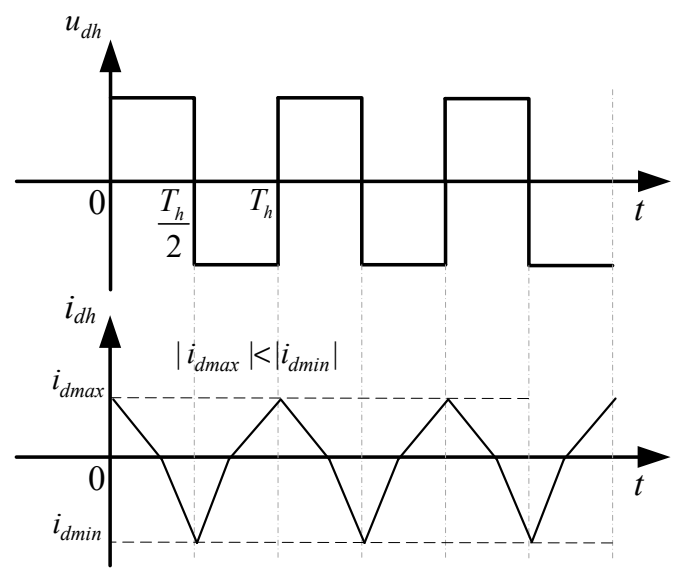

(b) $\Delta \theta=\pi$

Figure 4. Ideal $d$-axis current waveform at different $\Delta \theta$.

For the $d$-axis current, when the observed value of the position converges to the actual value, it can be expressed as in Equation (8).

$$
\frac{d i_{\hat{d} h}}{d t}=\frac{V_{h}(-1)^{k}}{\sum L-\Delta L}
$$

As the injection voltage increases, the fluctuation of the $d$-axis current becomes larger. The relationship between linear motor normal force and $d$-axis current can be approximated as in Equation (9) [29]

$$
\left\{\begin{array}{l}
F_{y} \propto\left(F_{d}+2 F_{f}\right)^{2} \\
F_{d} \propto i_{d}
\end{array}\right.
$$

where $F_{d}$ denotes the magnetomotive force of the $d$-axis, and $F_{f}$ denotes the magnetomotive force of the permanent magnet. Therefore, fluctuations in the $d$-axis current can cause fluctuations in the normal force of PMLSM which will affect the suspension system. 


\section{FIR Filter Analysis and Design}

\subsection{Current Frequency Spectrum Analysis}

Since the filter design was based on the frequency characteristics and the position was estimated using $i_{\hat{q}}$, the frequency characteristics of $i_{\hat{q}}$ needed to be analyzed. When using the space vector pulse width modulation (SVPWM) strategy to control the motor, it was necessary to modulate the calculated reference wave with a high-frequency carrier to generate a pulse-width modulating (PWM) signal to control the inverter. In this process, harmonic components related to the carrier wave are generated. When considering the influence of SVPWM harmonics, $i_{\hat{q}}$ can be expressed as:

$$
i_{\hat{q}}=i_{\hat{q} f}+i_{\hat{q} h}+i_{\hat{q} h m}
$$

where $i_{\hat{q} f}, i_{\hat{q} h}$, and $i_{\hat{q} h m}$ are a fundamental current, a high-frequency response current generated by the injection voltage, and a harmonic current generated by SVPWM, respectively.

When the motor runs stably, $i_{\hat{q} f}$ remains basically unchanged. So, $i_{\hat{q} f}$ can be regarded as a DC component. Even if the motor's operating state changes, $i_{\hat{q} f}$ changes much slower than $i_{\hat{q} h m}$ and $i_{\hat{q} h}$. Therefore, the frequency of $i_{\hat{q} f}$ can be approximately considered as $0 \mathrm{~Hz}$ [30].

The harmonics of SVPWM are mainly the sideband harmonics near the first and second carrier frequencies [31]. Considering the motor speed is low, it can be approximated that the frequency of $i_{\hat{q} h m}$ is concentrated on the first and second carrier frequencies.

It can be seen from Equation (6) that the frequency of $i_{\hat{q} h}$ is the same as that of the high-frequency injection signal. By using the Fourier transform, the frequency domain expression of the signal shown in Figure 2 can be incorporated into Equation (11), which indicates the frequency of $i_{\hat{q} h}$ is mainly the odd-numbered multiples of the injection signal.

$$
v(\omega)=b_{n} \sin \left(\frac{2 \pi n}{T_{h}} t\right)
$$

where $b_{n}=\left\{\begin{array}{c}0(n \text { is even number }) \\ \frac{4}{n \pi}(n \text { is odd number })\end{array}\right.$.

In this paper, the carrier frequency of SVPWM was $10 \mathrm{kHz}$, the injection signal frequency was $5 \mathrm{kHz}$, and the current sampling frequency was $50 \mathrm{kHz}$. The components less than the Nyquist frequency were considered. The frequency distribution of the three components in $i_{\hat{q}}$ are expressed in Table 1.

Table 1. Distribution of the components of $i_{\hat{q}}$.

\begin{tabular}{cc}
\hline Component & Frequency (kHz) \\
\hline$i_{\hat{q} f}$ & Approximate 0 \\
$i_{\hat{q} h m}$ & 10,20 \\
$i_{\hat{q} h}$ & 5,15 \\
\hline
\end{tabular}

\subsection{Principle of FIR Filter}

The essence of the FIR filter is to weigh and sum the input values of the past time. Compared with the IIR filter, there is no need to feedback the output, so the structure is simple and easy to implement in programming. The FIR filter can be described as:

$$
y[n]=\sum_{k=0}^{M} b_{k} x[n-k]
$$

where $y[n]$ is the filter output of the current moment, $x[n-k]$ is the system input of the $k$ sampling moment before the current moment, $M$ is filter order, and $b_{k}$ are the filter coefficients. 
As can be seen from Equation (12), the FIR filter uses the current input and the past $\mathrm{M}$ samples to calculate the system output. The filter coefficient $b_{k}$ represents the "weight" of the system input at different times. The determination of $b_{k}$ is the core of filter design.

When $b_{k}$ is determined, the frequency response of the FIR filter can be described as:

$$
H\left(e^{j \hat{\omega}}\right)=\sum_{k=0}^{M} b_{k} e^{-j \hat{\omega} k}
$$

where $\hat{\omega}=\omega T_{s}$ denotes the normalized angular frequency, and $\omega$ and $T_{s}$ are the actual angular frequency and sampling frequency, respectively. When the coefficients of the filter are even and symmetrical, approximately $M / 2$ (i.e., $b_{k}=b_{M-k}(\mathrm{k}=0,1,2, \cdots \mathrm{M} / 2)$ ), the FIR filter will be a linear phase filter. Using the Euler formula, Equation (13) can be derived as:

$$
H\left(e^{j \hat{\omega}}\right)=e^{-j \frac{M}{2} \hat{\omega}} A(\hat{\omega})
$$

where

$$
A(\hat{\omega})= \begin{cases}b_{\frac{M}{2}}+\sum_{k=0}^{\frac{M}{2}-1} 2 b_{k} \cos \hat{\omega}\left(\frac{M}{2}-k\right) & M=2,4,6,8 \cdots \\ \frac{M-1}{2} 2 b_{k} \cos \hat{\omega}\left(\frac{M}{2}-k\right) & M=1,3,5,7 \cdots \\ \sum_{k=0} 2 \ldots\end{cases}
$$

In Equation (15), $A(\hat{\omega})$ is a real number which has no influence on the phase of the output signal. The norm of $e^{-j \frac{M}{2} \hat{\omega}}$ is 1 which means it has no effect on the amplitude of the output signal. Therefore, the amplitude-frequency characteristic and phase-frequency characteristic of the frequency response function in Equation (14) can be expressed as in Equation (16).

$$
\left\{\begin{array}{c}
\left|H\left(e^{j \hat{\omega}}\right)\right|=|A(\hat{\omega})| \\
\angle H\left(e^{j \hat{\omega}}\right)=-\frac{M}{2} \hat{\omega}
\end{array}\right.
$$

It can be seen from the phase-frequency characteristic in Equation (16) that the time delay caused by the FIR filter is proportional to the filter order $M$. As the filter order decreases, the time delay caused by the filter also decreases. Therefore, it is necessary to reduce the order of the filter when the requirements are met.

\subsection{FIR Filter Design Method}

According to the signal processing flow illustrated in Figure $3, i_{\hat{q} h}$ needs to be removed at first. Therefore, at the frequency of $i_{\hat{q} h}$, the amplitude response of the designed filter should be as small as possible. So, it is better to change $b_{k}$ to make the amplitude response zero at the frequency of $i_{\hat{g} h}$. In Table 1 , since the frequencies of $i_{\hat{g} h}$ are mainly $5 \mathrm{kHz}$ and $15 \mathrm{kHz}$, the amplitude-frequency characteristics of the FIR filter should satisfy Equation (17):

$$
\left\{\begin{array}{l}
\left|H\left(e^{j \hat{\omega}_{5 k}}\right)\right|=0 \\
\left|H\left(e^{j \hat{\omega}_{15 k}}\right)\right|=0
\end{array}\right.
$$

where $\hat{\omega}_{5 k}=0.2 \pi$ and $\hat{\omega}_{15 k}=0.6 \pi$ denotes the normalized frequency of $5 \mathrm{kHz}$ and $15 \mathrm{kHz}$, respectively.

In Figure $3, i_{\hat{q} h}$ is regained by subtraction. To ensure the harmonic signals introduced by SVPWM are eliminated at the same time, the amplitude-frequency characteristics at the frequencies of $10 \mathrm{kHz}$ and $20 \mathrm{kHz}$ must be the same. Therefore, the amplitude-frequency response of the filter should also satisfy Equation (18).

$$
\left|H\left(e^{j \hat{\omega}_{10 k}}\right)\right|=\left|H\left(e^{j \hat{\omega}_{20 k}}\right)\right|
$$


where $\hat{\omega}_{10 k}=0.4 \pi$ and $\hat{\omega}_{20 k}=0.8 \pi$ denotes the normalized frequency of $10 \mathrm{kHz}$ and $20 \mathrm{kHz}$, respectively.

Combining Equations (17) and (18) with Equation (15) and (16), a system of equations in matrix form is obtained. When $M$ is an even number, it can be described as:

$$
\left[\begin{array}{cccc}
2 \cos \frac{M}{2} \hat{\omega}_{5 k} & \cdots & 2 \cos \hat{\omega}_{5 k} & 1 \\
2 \cos \frac{M}{2} \hat{\omega}_{15 k} & \cdots & 2 \cos \hat{\omega}_{15 k} & 1 \\
\operatorname{sub}\left(\frac{M}{2}\right) & \cdots & \operatorname{sub}(1) & 0
\end{array}\right]\left[\begin{array}{c}
b_{0} \\
\vdots \\
b_{\frac{M}{2}}
\end{array}\right]=0
$$

When $M$ is an odd number, it can be described as:

$$
\left[\begin{array}{cccc}
\cos \frac{M}{2} \hat{\omega}_{5 k} & \cdots & \cos 1.5 \hat{\omega}_{5 k} & \cos 0.5 \hat{\omega}_{5 k} \\
\cos \frac{M}{2} \hat{\omega}_{15 k} & \cdots & \cos 1.5 \hat{\omega}_{15 k} & \cos 0.5 \hat{\omega}_{15 k} \\
\operatorname{sub}\left(\frac{M}{2}\right) & \cdots & \operatorname{sub}(1.5) & \operatorname{sub}(0.5)
\end{array}\right]\left[\begin{array}{c}
b_{0} \\
\vdots \\
b_{\frac{M-1}{2}}
\end{array}\right]=0
$$

where $\operatorname{sub}(n)=\left|\cos n \hat{\omega}_{10 k}-\cos n \hat{\omega}_{20 k}\right| n=M / 2, M / 2-1, M / 2-2, \cdots(n>0)$.

Equations (19) and (20) are homogeneous linear equations. When the filter order $M$ is determined, unknown quantities are only $b_{k}$, the filter coefficients. When the rank of the coefficient matrix is smaller than the number of unknown quantities, the system of equations has a non-zero solution. When $M$ $=6$ or $M=7$, the number of unknown quantities is four. Since the maximum rank of the coefficient matrix of Equations (17) and (18) is three, the equations must have a non-zero solution. Therefore, the maximum filter order is only seven in this design. The solution process of the filter coefficients can be expressed with the flowchart in Figure 5 , and the obtained solution vector is $b_{k}$.

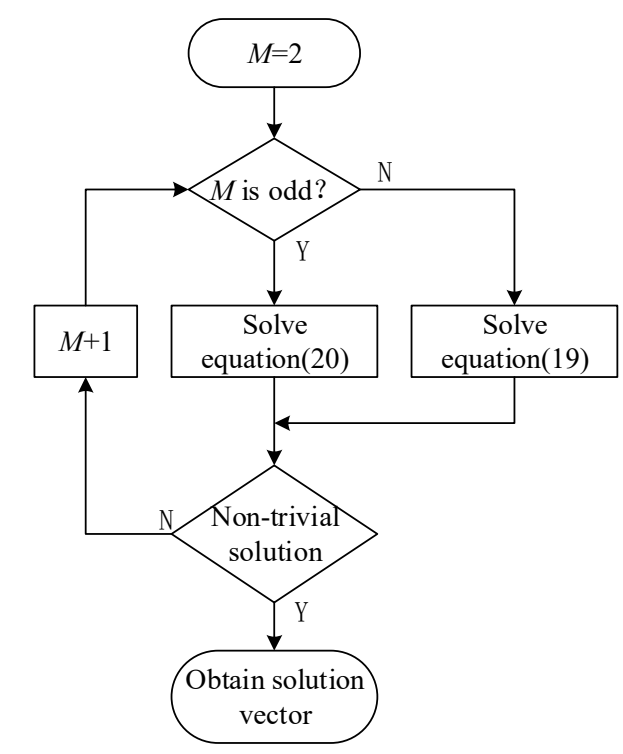

Figure 5. Solution process of filter coefficients.

According to this method, a non-zero solution appears when $M=5$, i.e. $b_{k}=[1,0,0,0,0,1]^{\mathrm{T}}$ and $M$ is less than seven, consistent with the theoretical analysis. This designed FIR filter can be described as in Equation (21).

$$
y[n]=x[n]-x[n-5]
$$

Its amplitude-frequency response curve and phase-frequency response curve are shown in Figure 6. 

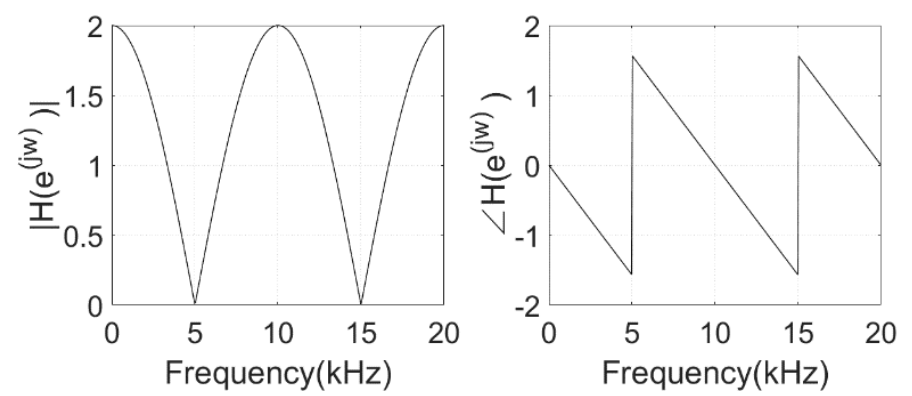

Figure 6. Amplitude-frequency response curve (left) and phase-frequency response curve (right).

From Figure 6, it can be seen that the amplitude-frequency response of the filter meets the expected requirements at $5 \mathrm{kHz}, 10 \mathrm{kHz}, 15 \mathrm{kHz}$, and $20 \mathrm{kHz}$. Since there is no phase shift at $10 \mathrm{kHz}$ and $20 \mathrm{kHz}$, the phase compensation can be omitted. In other words, the phase compensation is 0 in this design. Meanwhile, the amplitude gain of this filter at $10 \mathrm{kHz}$ and $20 \mathrm{kHz}$ is two. Thus, the filter output should be multiplied by 0.5 before subtraction in the process of extracting $i_{\hat{q} h}$.

\section{Simulation and Experiment Results}

In order to verify the effectiveness of the FIR filter designed in this paper, simulation and experimental verification were carried out. The frequency of injection signal and the switching frequency of inverter were $5 \mathrm{kHz}$ and $10 \mathrm{kHz}$, respectively. The topology of the inverter based on MOSFET was a three-phase full bridge, and the digital controller used for experiments was based on a TMS320F28335. The experiment platform is shown in Figure 7, and the parameters of PMLSM are in Tables 2 and A1.
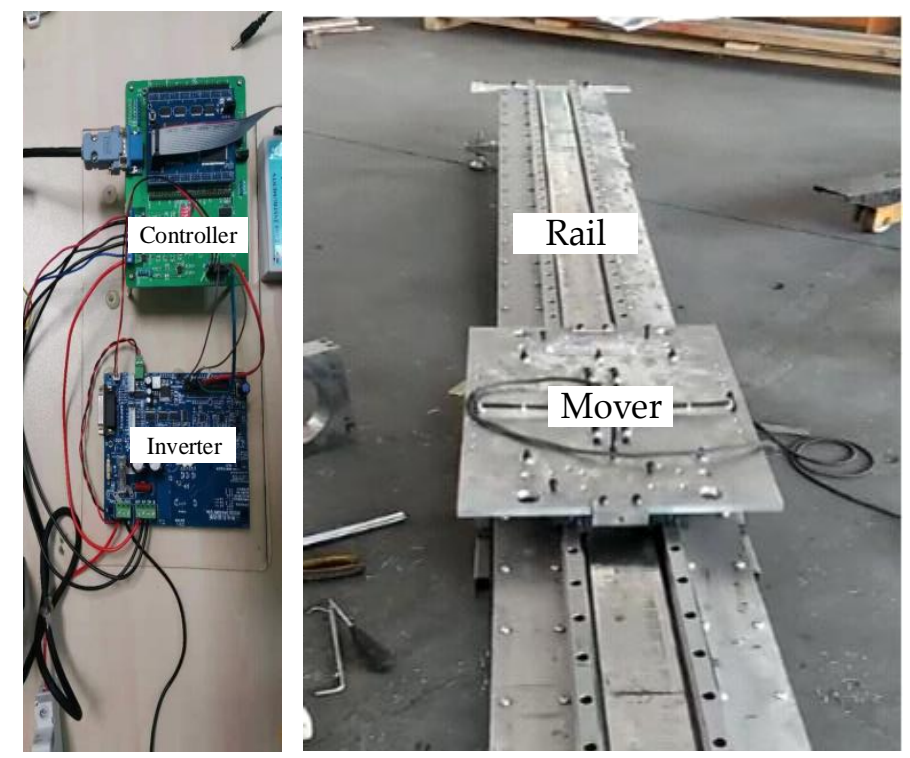

Figure 7. Experimental platform used for the test.

Table 2. Parameters of PMLSM.

\begin{tabular}{cc}
\hline Parameter & Value \\
\hline Resistance $(\Omega)$ & 1.3 \\
Inductance $(\mathrm{mH})$ & 7.8 \\
Continuous current $(\mathrm{A})$ & 7.5 \\
Pole pitch $(\mathrm{m})$ & 0.018 \\
Thrust constant $(\mathrm{N} / \mathrm{A})$ & 65.5 \\
Mover mass $(\mathrm{kg})$ & 5.8 \\
\hline
\end{tabular}


Figure 8 is the overall block diagram of the position sensorless control system. The observer in this control system is shown in Figure 3.

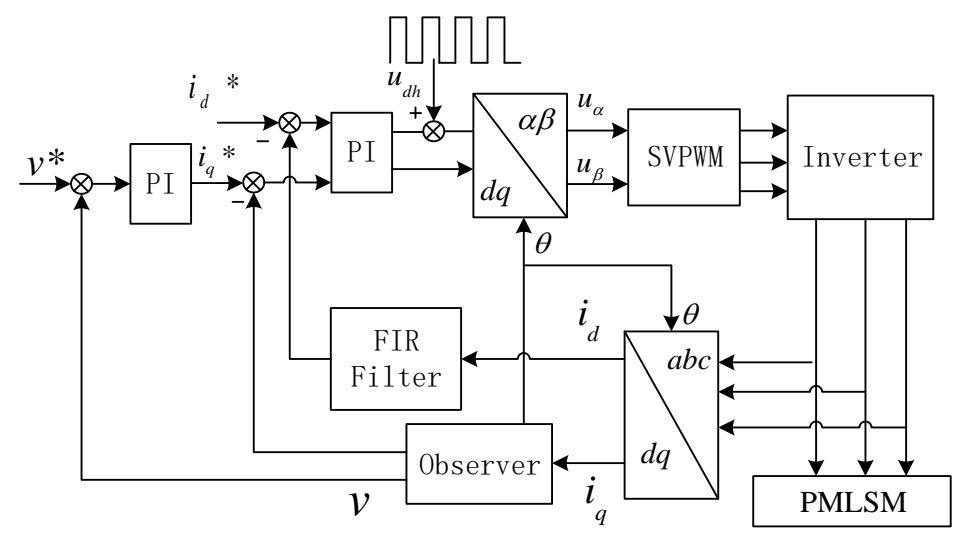

Figure 8. Block diagram of the position-sensorless control system based on high-frequency square-wave voltage injection.

With the help of the fast Fourier transform tool in MATLAB/Simulink, spectrum analysis of current is given. Figure 9 shows the frequency spectrum comparison of $i_{\hat{q}}$ before and after signal filtering in simulation which verifies the effect of FIR filter and signal separation. In PMLSM, $0 \mathrm{~Hz}, 10 \mathrm{kHz}$, and $20 \mathrm{kHz}$ components were the main interference in $q$-axis current, which is shown in Figure $9 \mathrm{a}$. After filtering, $5 \mathrm{kHz}$ and $15 \mathrm{kHz}$ components were eliminated, leaving only $0 \mathrm{~Hz}, 10 \mathrm{kHz}$, and $20 \mathrm{kHz}$ components. The amplitude and phase of $0 \mathrm{~Hz}, 10 \mathrm{kHz}$, and $20 \mathrm{kHz}$ components in original current and filtered current were the same. Therefore, by subtracting the filtered current from the original current, these components disappeared and only $5 \mathrm{kHz}$ and $15 \mathrm{kHz}$ components were left, which is shown in Figure $9 \mathrm{~b}$. Although there were other components besides $5 \mathrm{kHz}$ and $15 \mathrm{kHz}$ components in Figure $9 \mathrm{~b}$, the amplitude of these noise components was much smaller than that of $0 \mathrm{~Hz}, 10 \mathrm{kHz}$, and $20 \mathrm{kHz}$ components which means the main interference was eliminated. Therefore, the main goal was achieved, and the simulation results were consistent with the theoretical analysis.

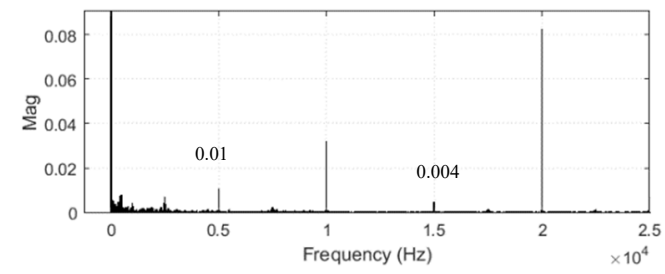

(a) before signal processing

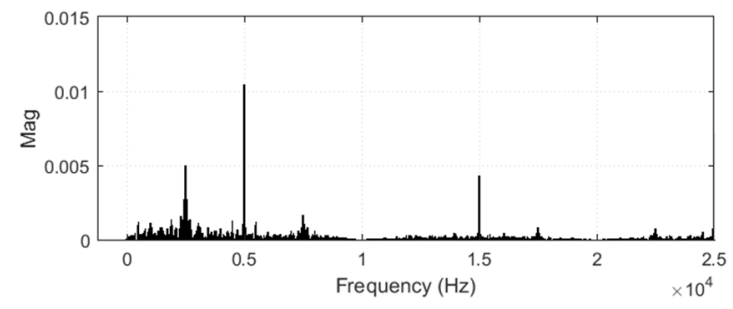

(b) after signal processing

Figure 9. Frequency spectrum comparison of $i_{\hat{q}}$ before and after signal processing.

In order to further verify the effectiveness of the designed filter, position estimation experiments were conducted on the experimental platform shown in Figure 7. After the experiment was over, the experiment results were exported for plotting which are shown in Figures 10-14.

The initial position of the magnetic pole was set to $6 \mathrm{~mm}$ ahead of A-phase axis, and the position observation was divided into two stages. At $0 \sim 0.3 \mathrm{~s}$, the initial position was estimated. After $0.3 \mathrm{~s}$, the motor started to move at low speed. The experiment results of position estimation after using this low-order FIR filter is shown in Figure 10. It can be seen that the estimated position value converged near the actual value. Therefore, the position estimation of PMLSM can be achieved by using this low-order FIR filter.

Figures 11-13 shows the results of magnetic pole position estimation under different conditions. In order to describe the error better, this paper used the absolute value of the ratio of deviation to pole 
pitch as the standard for measuring accuracy. In Figure 11, the position observation error exceeded $7 \%$ of the pole pitch with no filter used when the amplitude of the injection voltage was $50 \mathrm{~V}$. When the amplitude of the injection voltage increased to $100 \mathrm{~V}$, the position observation result is shown in Figure 12. The maximum error of the observation did not exceed $4 \%$ of the pole pitch, improving the observation accuracy. Figure 13 shows the observation result after adding the low-order FIR filter designed in this paper with an injection voltage of $50 \mathrm{~V}$. In this case, the maximum error was only slightly higher than $4 \%$ of the pole pitch. Therefore, with this low-order FIR filter, the position estimation accuracy can be improved to an approximate level compared with the method of increasing injection voltage amplitude.

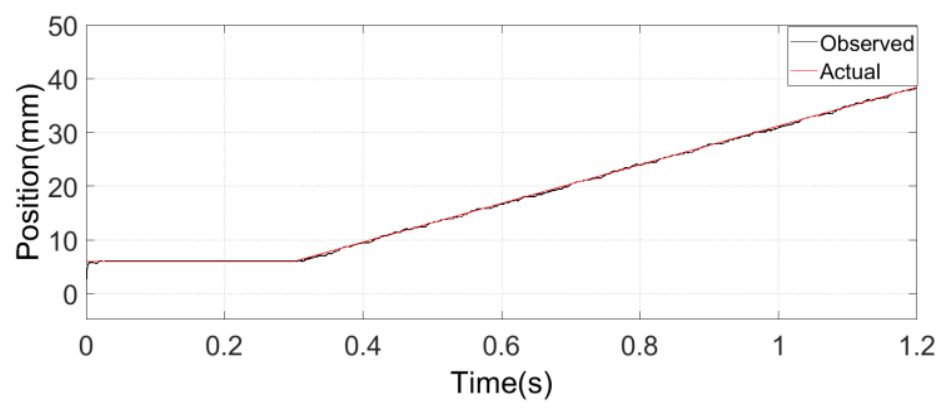

Figure 10. Position observation result at zero and low speed.
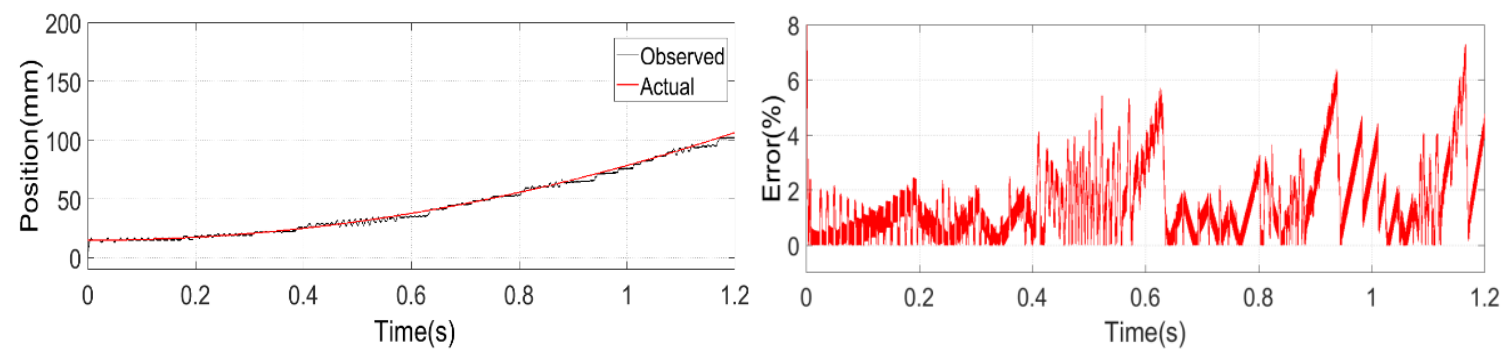

Figure 11. Position observation results at an injection voltage of $50 \mathrm{~V}$ without filter.
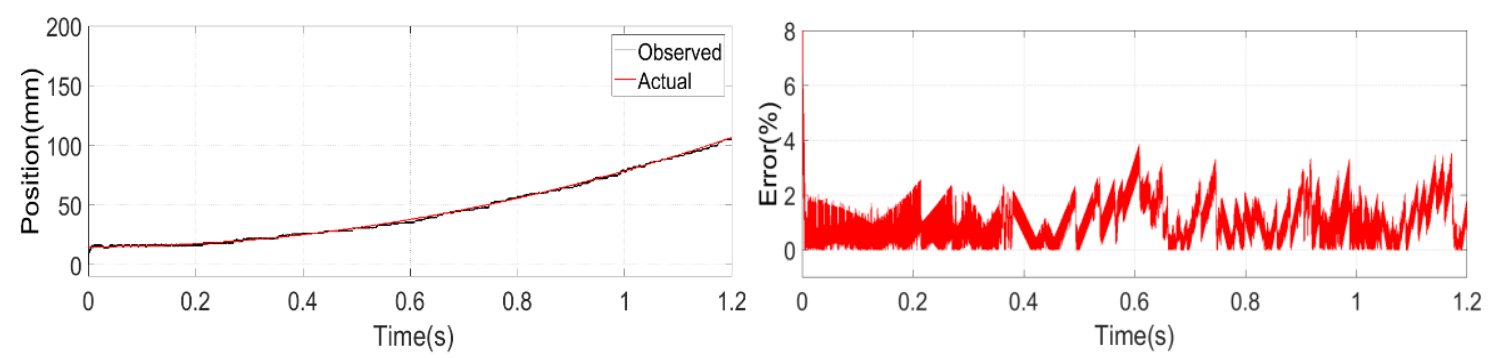

Figure 12. Position observation results at an injection voltage of $100 \mathrm{~V}$ without a filter.
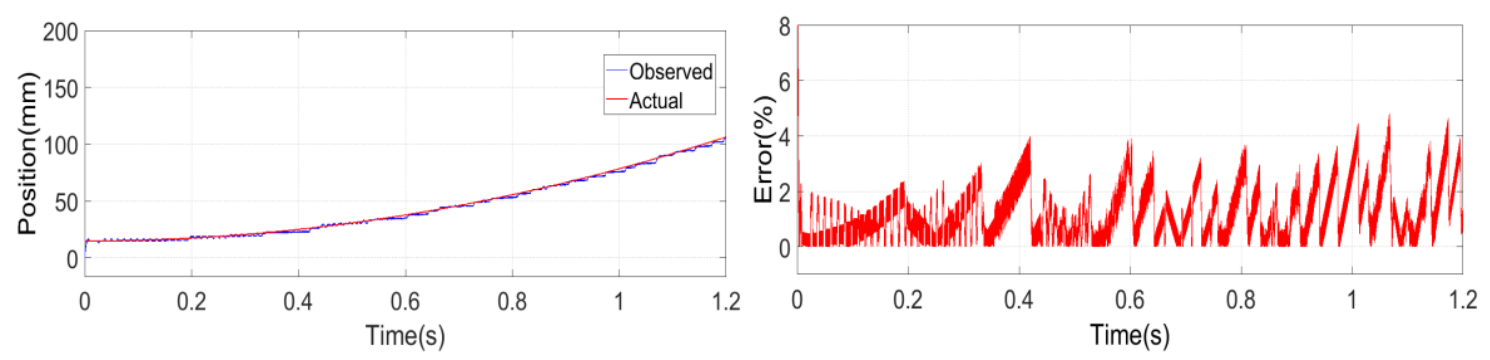

Figure 13. Position observation results at an injection voltage of $50 \mathrm{~V}$ with the FIR filter designed in this paper. 
In order to analyze the impact on the suspension system when the injection voltage amplitude is different, Figure 14 shows the $d$-axis current with an injection voltage of $50 \mathrm{~V}$ and $100 \mathrm{~V}$. It can be seen that the $d$-axis current fluctuation became significantly smaller when the injection voltage was $50 \mathrm{~V}$. From the analysis in Section 2, the $d$-axis current fluctuation was related to the normal force fluctuation. Smaller $d$-axis current fluctuation can reduce the fluctuation of normal force, thereby reducing the impact on the suspension system. Under the condition of ensuring observation accuracy, the FIR filter design method proposed in this paper can reduce the amplitude of injection voltage compared with the filterless method. Therefore, it can bring a smaller fluctuation of the $d$-axis current and normal force.

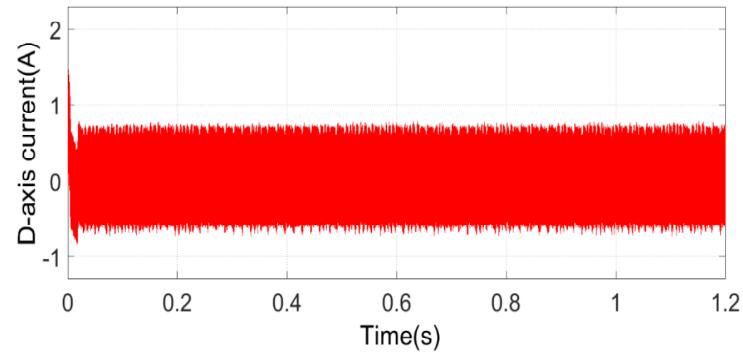

(a) The amplitude of injection voltage is $50 \mathrm{~V}$

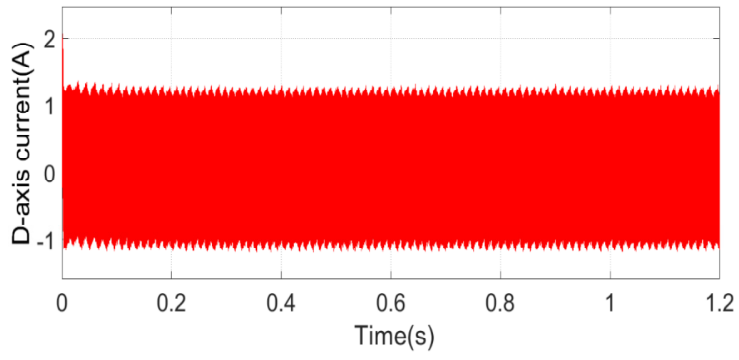

(b) The amplitude of injection voltage is $100 \mathrm{~V}$

Figure 14. The $d$-axis current at different amplitudes of injection voltage.

\title{
5. Conclusions
}

In the high-frequency signal injection method, digital filters were used to extract high-frequency response currents. This paper presented a design method of an FIR filter based on the frequency spectrum of motor current and the frequency response characteristics of the FIR filter. Through theoretical analysis, simulation, and experiment, the following conclusions can be drawn:

1. The time delay caused by the FIR filter was proportional to the filter order. It was necessary to reduce the order of the filter when the requirements are met;

2. By using the designed method proposed in this paper, a low-order FIR filter can be obtained. Its order was as low as five which was much lower than that of the FIR filter designed by the traditional method;

3. The low-order FIR filter designed in this study can complete the magnetic pole position estimation. Compared with the estimation system without filter, the application of this new filter can improve the position estimation accuracy without increasing injection voltage amplitude.

Author Contributions: Conceptualization, H.Z.; Formal analysis, H.Z. and L.Z.; Funding acquisition, L.Z. and C.Z.; Investigation, H.Z.; Methodology, C.Z.; Project administration, L.Z.; Resources, J.L.; Supervision, L.Z.; Validation, C.Z.; Writing-original draft, H.Z.; Writing-review and editing, J.C. and L.S. All authors have read and agreed to the published version of the manuscript.

Funding: The project presented in this article is supported by National Key R\&D Program of China (2016YFB1200601-B22).

Conflicts of Interest: The authors declare no conflict of interest.

\section{Nomenclature}

Abbreviation or Variable
PMLSM
PMSM
IIR
FIR
SNR
SRF
$i_{\hat{q} f}$

\author{
Definition \\ permanent magnet linear synchronous motor \\ permanent magnet synchronous motor \\ infinite impulse-response \\ finite impulse-response \\ signal-to-noise ratio \\ synchronization reference frame \\ fundamental current
}




$\begin{array}{ll}i_{\hat{q} h} & \text { high-frequency response current } \\ i_{\hat{q} h m} & \text { harmonic current } \\ V_{h} & \text { injection voltage amplitude } \\ \Delta \theta & \text { error between the estimated position and the actual position } \\ \Delta L & \text { half of the difference of } d-q \text { axis inductance } \\ \sum L & \text { half of the sum of } d-q \text { axis inductance } \\ \tau & \text { pole pitch } \\ p & \text { differential operator } \\ \psi_{f} & \text { flux linkage of the permanent magnet } \\ \hat{\theta} & \text { estimated position } \\ v & \text { motor speed } \\ M & \text { filter order } \\ b_{k} & \text { filter coefficient }\end{array}$

\section{Appendix A}

Table A1. Parameters of PMLSM.

\begin{tabular}{cc}
\hline Parameter & Value \\
\hline Resistance $(\Omega)$ & 1.3 \\
Inductance $(\mathrm{mH})$ & 7.8 \\
Continuous current $(\mathrm{A})$ & 7.5 \\
Pole pitch $(\mathrm{m})$ & 0.018 \\
Thrust constant $(\mathrm{N} / \mathrm{A})$ & 65.5 \\
Mover mass $(\mathrm{kg})$ & 5.8 \\
\hline
\end{tabular}

\section{References}

1. Song, X.; Dou, F.; Dai, C. Modeling and Design of the Speed and Location System for Low Speed Maglev Vehicle. In Proceedings of the Fifth ICICTA, Zhangjiajie, China, 12-14 January 2012; pp. 205-209.

2. Zhang, D.; Long, Z.; Dai, C. Design and realization of a novel position-and-speed measurement system with communication function for the low-speed maglev train. Sens. Actuators A Phys. 2013, 203, 261-271. [CrossRef]

3. Dai, C.; Huang, C.; Tan, L. Comparison and Analysis of Signal Processing Methods for Induction Loop-Cable Position and Speed Detection System. In Proceedings of the 2018 Chinese Automation Congress (CAC), Xi'an, China, 30 November-2 December 2018; pp. 2873-2878.

4. Maeda, T.; Watanabe, K.; Ono, M. Train position detecting system using radio millimeter-waves. In Proceedings of the 10th International Conference on Computer System Design and Operation in the Railway and Other Transit Systems, Prague, Czech Republic, 10-12 July 2006; pp. 469-476.

5. Yufeng, L.; Tefang, C.; Shu, C. Processing and Fusion of Velocity and Position Signal of Medium and High Speed Maglev Train. In Proceedings of the 13th World Congress on Intelligent Control and Automation (WCICA), Changsha, China, 4-8 July 2018; pp. 1578-1583.

6. Wang, H.; Ge, X.; Liu, Y. Second-Order Sliding-Mode MRAS Observer-Based Sensorless Vector Control of Linear Induction Motor Drives for Medium-Low Speed Maglev Applications. IEEE Trans. Ind. Electron. 2018, 65, 9938-9952. [CrossRef]

7. Shen, J.X.; Zhu, Z.Q.; Howe, D. Improved speed estimation in sensorless PM brushless AC drives. IEEE Trans. Ind. Appl. 2002, 38, 1072-1080. [CrossRef]

8. Li, Y.; Zhu, Z.Q.; Howe, D.; Bingham, C.M. Improved Rotor Position Estimation in Extended Back-EMF Based Sensorless PM Brushless AC Drives with Magnetic Saliency. In Proceedings of the 2007 IEEE International Electric Machines \& Drives Conference, Antalya, Turkey, 3-5 May 2007; pp. 214-219.

9. Kim, H.; Son, J.; Lee, J. A High-Speed Sliding-Mode Observer for the Sensorless Speed Control of a PMSM. IEEE Trans. Ind. Electron. 2011, 58, 4069-4077.

10. Quang, N.K.; Hieu, N.T. FPGA-based sensorless PMSM speed control using reduced-order extended Kalman filters. IEEE Trans. Ind. Electron. 2014, 61, 6574-6582. [CrossRef] 
11. Raca, D.; Garcia, P.; Reigosa, D.; Briz, F.; Lorenz, R. A comparative analysis of pulsating vs. rotating vector carrier signal injection-based sensorless control. In Proceedings of the Twenty-Third Annual IEEE APEC, Austin, TX, USA, 24-28 February 2008; pp. 879-885.

12. Luo, X.; Tang, Q.; Shen, A.; Zhang, Q. PMSM Sensorless Control by Injecting HF Pulsating Carrier Signal Into Estimated Fixed-Frequency Rotating Reference Frame. IEEE Trans. Ind. Electron. 2016, 63, 2294-2303. [CrossRef]

13. Liu, J.M.; Zhu, Z.Q. Novel Sensorless Control Strategy With Injection of High-Frequency Pulsating Carrier Signal Into Stationary Reference Frame. IEEE Trans. Ind. Appl. 2014, 50, 2574-2583. [CrossRef]

14. Tang, Q.; Shen, A.; Luo, X.; Xu, J. PMSM Sensorless Control by Injecting HF Pulsating Carrier Signal Into ABC Frame. IEEE Trans. Power Electr. 2017, 32, 3767-3776. [CrossRef]

15. Tang, Q.; Shen, A.; Luo, X.; Xu, J. IPMSM Sensorless Control by Injecting Bidirectional Rotating HF Carrier Signals. IEEE Trans. Power Electr. 2018, 33, 10698-10707. [CrossRef]

16. Ni, R.; Xu, D.; Blaabjerg, F.; Lu, K.; Wang, G.; Zhang, G. Square-Wave Voltage Injection Algorithm for PMSM Position Sensorless Control with High Robustness to Voltage Errors. IEEE Trans. Power Electr. 2017, 32, 5425-5437. [CrossRef]

17. Xu, P.L.; Zhu, Z.Q. Novel Square-Wave Signal Injection Method Using Zero-Sequence Voltage for Sensorless Control of PMSM Drives. IEEE Trans. Ind. Electron. 2016, 63, 7444-7454. [CrossRef]

18. Bian, Y.J.; Guo, X.H.; Song, X.F.; Wu, Y.F.; Chen, Y. Initial rotor position estimation of PMSM based on high-frequency signal injection. In Proceedings of the 2014 ITEC Asia-Pacific, Beijing, China, 31 August-3 September 2014; pp. 1-4.

19. Li, M.Q.; Wang, L. An Improved Low Speed Sensorless Control Strategy for Permanent Magnet Synchronous Motor. Trans. China Electrotech. Soc. 2018, 33, 1967-1974.

20. Li, Q.; Li, C.F.; Liu, K.; Huang, Y.Q. Digital filters design for sensorless control of PMSM. Electr. Mach. Control 2018, 8, 47-53.

21. Ji, W.; Shi, G.D.; Xu, B. An improved rotating HF signal injection method based on FIR filters for state estimation of BPMSM sensorless control. Adv. Mech. Eng. 2017, 9, 1-10. [CrossRef]

22. Li, H.; Zhang, X.; Xu, C.; Hong, J. Sensorless Control of IPMSM Using Moving-Average-Filter Based PLL on HF Pulsating Signal Injection Method. IEEE Trans. Energy. Convers. 2020, 35, 43-52. [CrossRef]

23. Kim, S.H.; Park, N.C. Simple sensorless algorithm for interior permanent magnet synchronous motors based on high-frequency voltage injection method. IET Electr. Power Appl. 2014, 8, 68-75.

24. Zhang, G.Q.; Wang, G.L.; Xu, D.G. Filterless Square-Wave Injection Based Initial Position Detection for Permanent Magnet Synchronous Machines. Trans. China Electrotech. Soc. 2017, 32, 162-168.

25. Zhang, X.; Li, H.; Yang, S.; Ma, M. Improved Initial Rotor Position Estimation for PMSM Drives Based on HF Pulsating Voltage Signal Injection. IEEE Trans. Ind. Electron. 2018, 65, 4702-4713. [CrossRef]

26. Kim, S.; Im, J.; Song, E.; Kim, R. A New Rotor Position Estimation Method of IPMSM Using All-Pass Filter on High-Frequency Rotating Voltage Signal Injection. IEEE Trans. Ind. Electron. 2016, 63, 6499-6509. [CrossRef]

27. Chen, S.; Chiang, H.; Liu, T.; Chang, C. Precision Motion Control of Permanent Magnet Linear Synchronous Motors Using Adaptive Fuzzy Fractional-Order Sliding-Mode Control. IEEE ASME Trans. Mech. 2019, 24, 741-752. [CrossRef]

28. Yang, S.; Yang, S.; Hu, J. Design Consideration on the Square-Wave Voltage Injection for Sensorless Drive of Interior Permanent-Magnet Machines. IEEE Trans. Ind. Electron. 2017, 64, 159-168. [CrossRef]

29. Lan, Y.P.; Hu, X.C.; Chen, Q.L. Finite Element Analysis of Electromagnetic Characteristics of Controllable Excitation Magnetic Suspension Feed Platform. CJME 2017, 53, 184-189. [CrossRef]

30. Yoon, Y.; Sul, S. Sensorless Control for Induction Machines Based on Square-Wave Voltage Injection. IEEE Trans. Power Electr. 2014, 29, 3637-3645. [CrossRef]

31. Liang, W.; Wang, J.; Luk, P.C.; Fang, W.; Fei, W. Analytical Modeling of Current Harmonic Components in PMSM Drive with Voltage-Source Inverter by SVPWM Technique. IEEE Trans. Energy Convers. 2014, 29, 673-680. [CrossRef]

(C) 2020 by the authors. Licensee MDPI, Basel, Switzerland. This article is an open access article distributed under the terms and conditions of the Creative Commons Attribution (CC BY) license (http://creativecommons.org/licenses/by/4.0/). 